\title{
Análisis constructivo y estructural de la catedral de Valencia
}

\section{Constructive and structural analysis of the cathedral of Valencia}

\author{
V. Llopis Pulido $^{(*)}$, A. Alonso Durá ${ }^{(*)}$, E. Fenollosa ${ }^{(*)}$, A. Martínez Boquera ${ }^{(*)}$
}

\section{RESUMEN}

Las características constructivas de la catedral de Valencia la convierten en un ejemplo representativo del gótico mediterráneo: reducida esbeltez, escasa diferencia de altura entre las naves y limitadas dimensiones de huecos.

Estas características se explican a partir del presente trabajo donde se muestra un estudio constructivo y un análisis del sistema estructural frente a cargas gravitatorias y efectos sísmicos. En el análisis se utiliza el método por empujes incrementales (pushover) y el método no-lineal de modelo de daño. La función de arbotantes y diafragmas quedará patente con la modificación del modelo MEF que eliminará progresivamente cada uno de estos elementos constructivos.

Los resultados muestran una suficiente capacidad resistente frente a un sismo con un período de retorno de 475 años que empeora si se considera de 950 años. Se evidenciará la necesidad de la continuidad muraria a través de los arbotantes que se convierten en auténticos muros diafragmáticos.

Palabras clave: Gótico mediterráneo; análisis sísmico; pushover; modelo de daño; elementos finitos; período retorno.

\section{ABSTRACT}

The cathedral of Valencia constructive characteristics make it a representative example of the Mediterranean Gothic: low slenderness, little height difference between the naves and limited voids dimension.

These differences are explained from the present work, where a constructive study and a structural system analysis under gravity loads and seismic effects are shown. For the analysis a pushover method and a non-linear damage model have been used. The buttresses and diaphragms function will be patent with the Finite Element structural model modification which will progressively remove each one of these constructive elements.

The results display a resistant capacity enough to face a seism of 450 years that gets worse if a return period of 950 years is considered. The need of the walls continuity trough the buttresses that turn into authentic diaphragm walls will be demonstrated.

Keywords: Mediterranean Gothic; seismic analysis; pushover; damage model; finite element; return period.

${ }^{(*)}$ Departamento Mecánica de los Medios Continuos y Teoría de Estructuras. Universitat Politècnica de València. Camino de Vera, s/n. 46022 Valencia, Spain.

Persona de contacto/Corresponding author: vllopis@mes.upv.es (V. Llopis Pulido)

Cómo citar este artículo/Citation: Llopis Pulido, V., Alonso Durá, A., Fenollosa, E., Martínez Boquera, A. (2016). Análisis constructivo y estructural de la catedral de Valencia. Informes de la Construcción, 68(543): e158, doi: http://dx.doi.org/10.3989/ic.15.102.

Licencia / License: Salvo indicación contraria, todos los contenidos de la edición electrónica de Informes de la Construcción se distribuyen bajo una licencia de uso y distribución Creative Commons Attribution License (CC BY) Spain 3.o. 


\section{INTRODUCCIÓN}

El sistema estructural de las grandes catedrales góticas centroeuropeas está formado por bóvedas de crucería que permiten concentrar las cargas gravitatorias sobre cuatro columnas. El muro pierde su función portante y se convierte en grandes vitrales que permiten la entrada de luz en el edificio (1).

La estructura de la catedral de Valencia se aparta de los cánones constructivos del gótico centroeuropeo, se trata de un ejemplo representativo de la denominada arquitectura gótica mediterránea (2), (3). Arquitectura que se caracteriza por su escasa esbeltez y por una disminución de la superficie destinada a vidriera, debido a la pequeña diferencia de altura entre la nave principal y las laterales (4). Distintas hipótesis han intentado justificar el porqué de estas diferencias, desde motivos relacionados con el clima, cuestiones compositivas, el carácter defensivo de este tipo de edificación, etc.

Recientes estudios relacionan estas peculiaridades del gótico mediterráneo con la geografía en que se ubican. En concreto al superponer su localización con el mapa sísmico de Europa se observa que se sitúan en zonas de mayor riesgo sísmico (5).

El presente trabajo mostrará el estudio constructivo de la catedral de Valencia y su análisis estructural, a partir de un modelo informático con E.F. (6). La manipulación de este modelo mediante la eliminación selectiva de arbotantes, diafragmas y/o refuerzos, genera cinco variaciones, cuyo análisis mostrará la función de cada uno de estos elementos estructurales.

El análisis estructural de la catedral, que contempla las acciones sísmicas además de las cargas gravitatorias, se realizará mediante el programa de E.F. en régimen no lineal ANGLE (7). Los efectos sísmicos se evaluarán mediante el método de análisis por empujes incrementales (pushover) (8) y el comportamiento de los materiales mediante el denominado «modelo de daño» (9).

Los resultados obtenidos proporcionarán un excelente conocimiento del comportamiento estructural y de los sistemas constructivos utilizados en el gótico mediterráneo.

\section{DESCRIPCIÓN}

La iglesia catedral basílica metropolitana de Valencia (1262) se sitúa sobre la antigua mezquita mayor musulmana. Es un edificio de planta de cruz latina con marcado crucero, formada en su origen por tres naves y tres crujías transversales.

La nave central es de tramos cuadrados mientras que las laterales son de tramos rectangulares con el eje mayor según el eje longitudinal del templo, delimitadas por una serie de capillas que configuran el espacio central. Su construcción se inició por la girola, de tramos pentagonales delimitados por un tramo de la capilla mayor y por dos capillas absidiales. Estas capillas se configuran radialmente, con una disposición poco común al considerar a eje de simetría un pilar y no una capilla.

En 1458 se realiza la ampliación de la catedral: se añade una cuarta crujía y se unen al templo los volúmenes de la capilla del Santo Cáliz y el campanario conocido como El Miguelete. Al final del siglo xvir se amplían capillas con cúpulas barrocas que configuran el estado actual (Figura 1a).

El cimborrio es de planta octogonal, se sitúa en el tramo central del crucero, articulado en dos cuerpos muy esbeltos y sin estribos, estas características lo han convertido en una obra que ha generado gran admiración (Figura 1b).

La sección transversal (Figura 2a) refleja las características que alejan a la catedral de Valencia de los ideales de proporción del gótico centroeuropeo. Por un lado la escasa diferencia de altura entre la nave principal y las naves laterales lo que supone una reducción de la superficie de las vidrieras. Por otro lado destacar la posición de los arbotantes por encima del empuje de la bóveda, situación que pone en duda su función estructural.

El sistema primario lo constituyen dos conjuntos de murosdiafragma, los muros longitudinales de la nave central y los transversales de cada tramo (Figura 2b), el conjunto configura una caja rígida de muros de gran potencia. El muro diafragmático transversal es perforado por los arcos de la nave central y las laterales, y por un arco de medio punto que permite el recorrido por la cubierta. El arbotante forma parte del diafragma, en realidad es el resultado de su perforación por el mencionado arco.
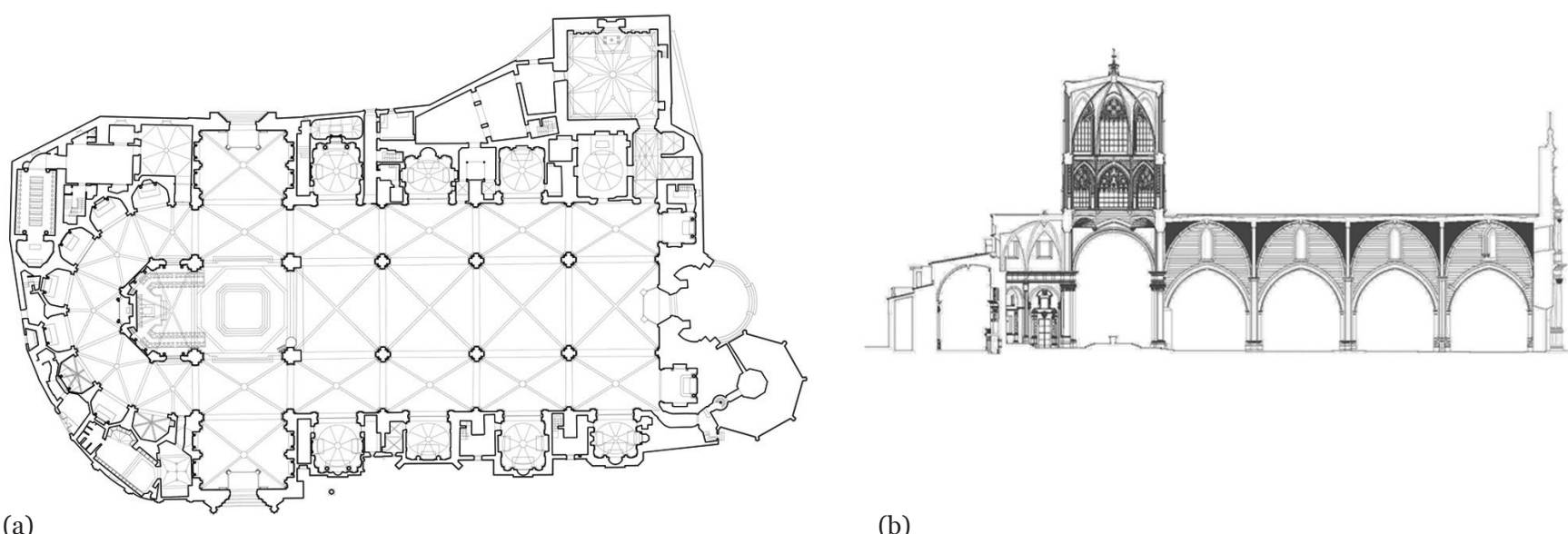

(b)

Figura 1. Catedral de Valencia: a) planta; b) sección longitudinal. (Planos autor Ramiro Moya). 


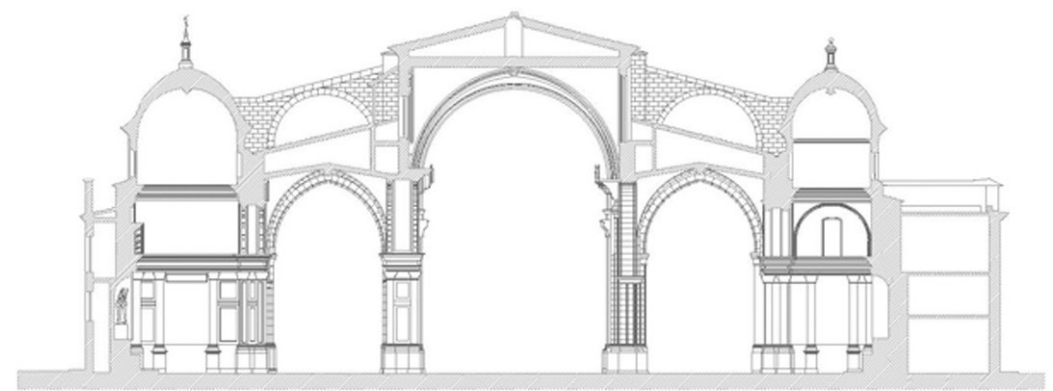

(a)

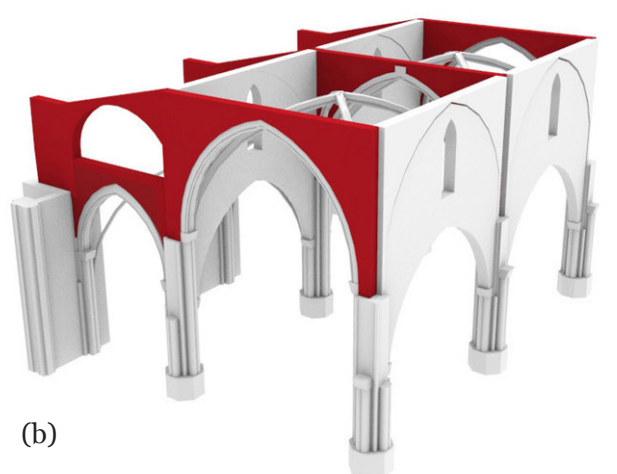

(b)

Figura 2. Catedral de Valencia: a) sección transversal (Plano autor Ramiro Moya); b) esquema estructural.

\section{ANÁLISIS CONSTRUCTIVO}

Para conocer en profundidad el sistema estructural de la catedral resulta fundamental hacer referencia al sistema constructivo de las iglesias denominadas «iglesias de reconquista» (10). Se trata de un sistema heredado de la tradición romana, en el que las iglesias están construidas con arcos diafragma transversalmente a la nave y que soportan la cubierta a dos vertientes, generalmente de madera y vista desde el interior. El origen del sistema se halla en las abadías cistercienses de los países mediterráneos, utilizado para cubrir las dependencias monásticas. Posteriormente este sistema se traslada a la construcción de iglesias donde se realizan modificaciones como la introducción del ábside y las capillas abovedadas.

En el caso de la catedral de Valencia y de muchas otras situadas en la franja del Mediterráneo, el sistema diafragmático queda enmascarado por las bóvedas que, para garantizar la durabilidad sustituyen a la cubierta de madera. En definitiva los arcos diafragmáticos proporcionan la estabilidad tramo a tramo, mientras que las bóvedas garantizan la durabilidad y solidez de la cubierta, además de conseguir un espacio bajo un mismo estilo.

La forma más elemental de bóveda de crucería es la que resulta de la intersección de dos bóvedas de cañón (en este caso de sección apuntada Figura 3). En sus aristas se concentran las tensiones como consecuencia de la pérdida de continuidad de su curvatura, estas discontinuidades tienen que ser reforzadas por medio de los nervios. Como las cargas gravitatorias se transmiten directamente a los nervios, el arco fajón aparentemente está exento de carga, por lo que se podría suponer que no son necesarios, ya que no desempeñan una función determinante para la estructura. Como hemos comentado en la catedral de Valencia se disponen potentes muro-diafragmas, por tanto, si estos muros no son fundamentales frente a cargas gravitatorias, las preguntas a plantearse son: ¿̇por qué razón se construyen?, ¿qué función desempeñan?

Las investigaciones realizadas por la profesora Cassinello plantean otras preguntas que desde un punto de vista diferente convergen en las mismas conclusiones. Según Cassinello la respuesta se obtiene al superponer el mapa sísmico de Europa y el mapa de localización de las catedrales góticas españolas (11). Se observa que aquellas iglesias con una geometría y unas proporciones que se alejan del modelo gótico tradicional se sitúan en zonas donde hay mayor riesgo sísmico. Por tanto el sismo justifica las diferencias entre las proporciones características del estilo gótico centroeuropeo y las del mediterráneo. Las particularidades de la catedral de Valencia, en relación a sus proporciones y a la ineficacia estructural de los arbotantes frente a cargas gravitatorias, quedan justificadas en la estructura pétrea oculta bajo su terraza.

Otro ejemplo que reproduce el sistema del gótico mediterráneo es la catedral de Tortosa, donde los potentes diafragmas presentan huecos para circulación pero de proporciones más reducidas que los de la catedral de Valencia. También se podrían citar la catedral de Sevilla, con diafragmas vistos desde el exterior, o la iglesia de Santa María del Mar de Barcelona.

\subsection{Toma de datos, interpretación constructiva}

Para llevar a cabo el análisis estructural de la catedral ha sido fundamental realizar una toma de datos exhaustiva que permita conocer el estado actual, para lo que se han anali-
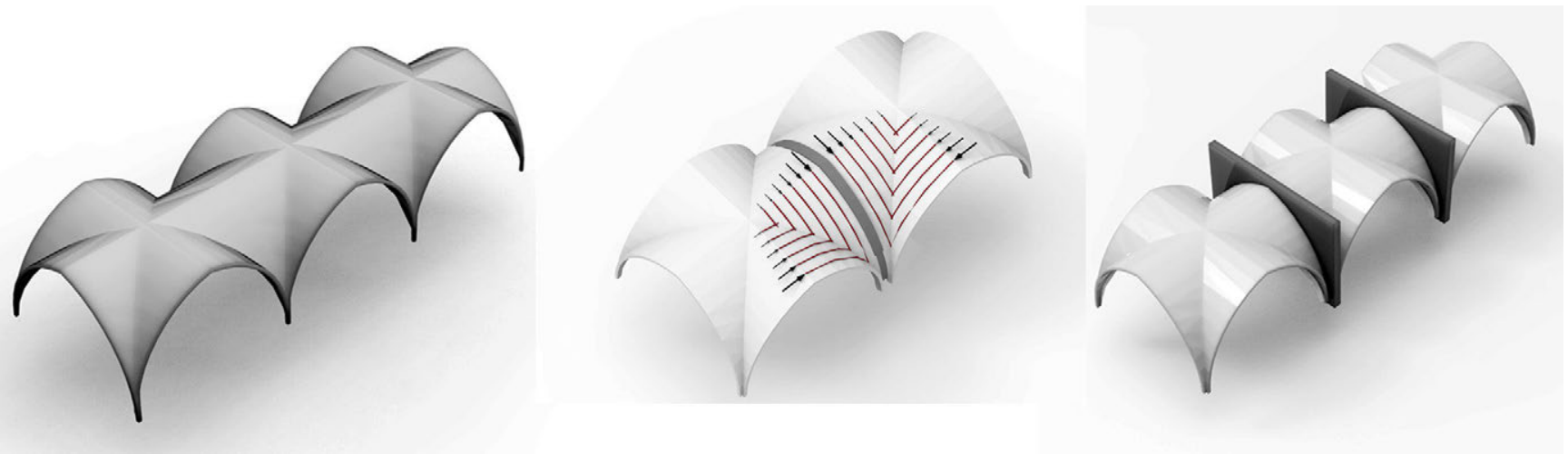

Figura 3. Esquema estructural de las bóvedas de la nave central de la catedral de Valencia. 
zado aquellas intervenciones de carácter estructural y constructivo. De todas estas actuaciones la más relevante fue la realizada por Ramiro Moya en 1976. En ella se eliminan las cubiertas inclinadas (Gilabert, 1773) y se recuperan las primitivas terrazas planas que cubren la nave principal y las laterales (Figura 4). Estas cubiertas originales están formadas por tabiques de ladrillo de $15 \mathrm{~cm}$ de espesor, separados 40 $\mathrm{cm}$, apoyadas directamente sobre las bóvedas. El espesor de la bóveda central es de pie y medio $(29+15 \mathrm{~cm})$, y las laterales de un pie $(29 \mathrm{~cm})$. Sobre los tabiques apoyan baldosas de $40 \mathrm{~cm}$ de longitud sujetas por una mezcla de cal, yeso y arena.

Para solucionar los daños provocados por las continuas filtraciones se aisló la cubierta por medio de una lámina impermeabilizante y se construyó una losa de hormigón armado de
$10 \mathrm{~cm}$ de espesor. Los ladrillos originales que pertenecían a la cubierta plana fueron recuperados y colocados posteriormente como remate final sobre la losa. En coronación de los muros se realizó un zunchado perimetral con un nervio de hormigón armado, y otros zunchos perpendiculares sobre los diafragmas de mampostería, en continuidad con los arcos fajones, además se consolidaron las grietas de los arbotantes.

Con la información recopilada y con el levantamiento geométrico a partir del escáner láser (12) se ha elaborado un modelo 3d que reproduce el sistema constructivo de la cubierta de la catedral. En la figura 5 a se observa el entramado murario con los diafragmas, la continuidad con los arbotantes, la disposición y tipo de bóvedas, en la figura $5 \mathrm{~b}$ se muestra la distribución de los tabiquillos sobre las bóvedas.

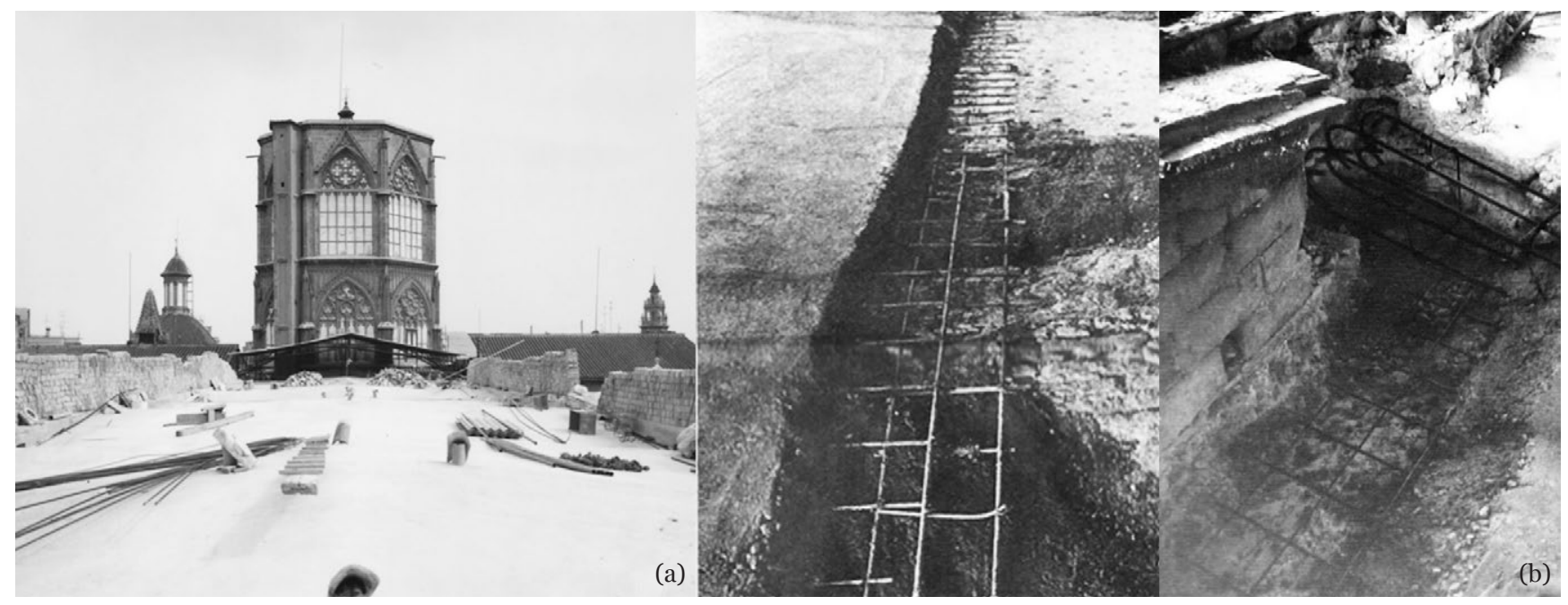

Figura 4. Intervención: a) cubierta original; central: losa de hormigón armado; b) zuncho hormigón armado sobre muros. (Fotos cedidas por Tirso de Ávila).

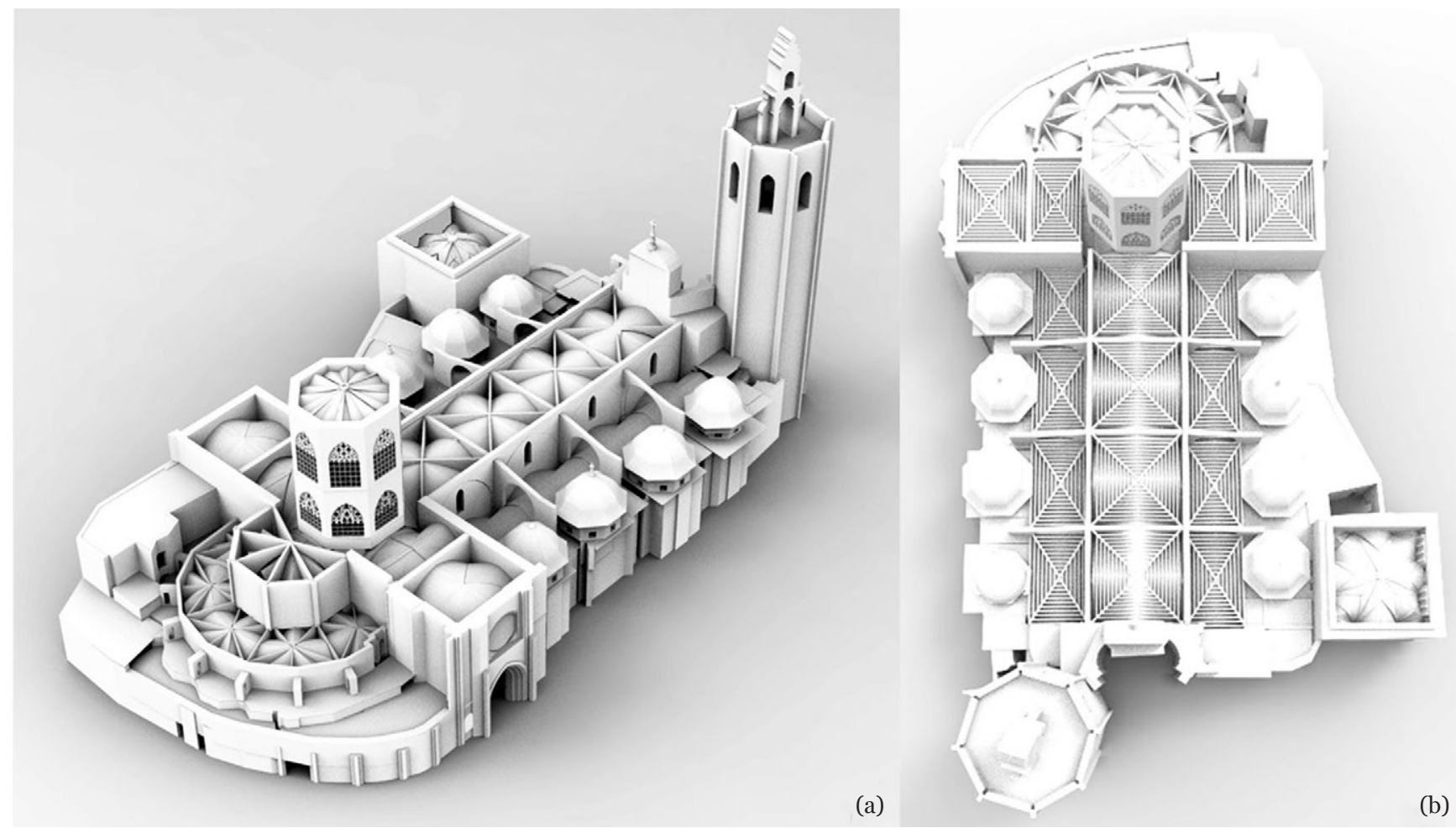

Figura 5. Interpretación cubierta: a) entramado murario, bóvedas y tabiques sobre nervios; b) disposición y orientación de los tabiquillos. 


\section{ANÁLISIS NUMÉRICO}

Por medio del análisis estructural es posible demostrar la capacidad estructural de los diafragmas para recibir los esfuerzos horizontales y estudiar su influencia en la mejora del comportamiento sísmico de la catedral. Además de analizar y comparar la efectividad del resto de elementos constructivos por medio de diferentes modelos de cálculo.

El análisis de elementos finitos se realiza por medio del software ANGLE, a partir de la geometría real y en base a su configuración constructiva se generan los modelos de cálculo con herramientas $\mathrm{CAD}$. Para evaluar el comportamiento sísmico de la catedral se utiliza el análisis no lineal pushover (13). El método empleado para simular el comportamiento de la piedra y el ladrillo es el «modelo de daño», uno de los métodos más rigurosos para reproducir la respuesta de los materiales frágiles.

\subsection{Propiedades mecánicas de los materiales}

Se han considerado dos tipos de materiales, la sillería para pilares y muros y el ladrillo para las bóvedas y para el relleno del núcleo central de pilares (14), (15). Para establecer las propiedades mecánicas se han considerado los ensayos realizados en los sillares del puente de la Trinidad de Valencia (16), ya que la procedencia de esta piedra es la misma que la de la catedral. Los resultados de estos ensayos se han corregido ligeramente al alza para tener en cuenta que las probetas se tomaron de sillares situados en el exterior, sometidos durante siglos a la degradación medioambiental y perdiendo densidad. En el análisis estructural realizado se han utilizado valores conservadores del lado de la seguridad, distinguiendo entre cuatro materiales (17), (18) (Tabla 1).

\subsection{Modelo constitutivo: modelo de daño}

La mecánica de daño introduce cambios a nivel microestructural del material a través de variables internas. Estas variables modifican la influencia de la historia del comportamiento del material en la evolución de las tensiones. La aparición de fisuras y la evolución de las mismas en el tiempo se describen como las trayectorias de los puntos que están dañados.

La fractura queda representada como un efecto de daño local que se puede caracterizar en función de la resistencia a compresión y a tracción del material, de los parámetros conocidos del material y de las funciones que controlan la evolución de la fisuración según el estado sucesivo de las tensiones en cada uno de los puntos.

El análisis estructural se ha realizado mediante el programa de Elementos Finitos en régimen no lineal ANGLE, mediante una aplicación del modelo de daño isótropo (19). El modelo de daño empleado se basa en la diferencia de comportamiento en compresión y tracción de este tipo de materiales, en la degradación de su rigidez por el nivel tensional y en el efecto en la respuesta en función del tamaño de la malla empleada.

La degradación de una zona del material que tiene un nivel de deterioro se representa mediante «huecos». El índice $d$ [1] indica el grado de deterioro del material $(0 \leq d \leq 1)$, siendo el valor o el estado no dañado y la unidad el deterioro total del área resistente.

$$
d=\frac{S-S^{\prime}}{S}
$$

$S$ representa la superficie total y $S^{\prime}$ el área resistente efectiva. La diferencia $S-S^{\prime}$ expresa la superficie de huecos que se han producido en el material. La relación entre la tensión de Cauchy $\sigma^{\prime}$ y la tensión efectiva que actúa sobre la sección resistente efectiva $(\sigma)$, es calculada por la condición de equilibrio

$$
\sigma=(1-d) \sigma^{\prime}=(1-d) \mathrm{E} \varepsilon
$$

Este índice escalar es suficiente para representar correctamente el comportamiento de materiales como las fábricas de ladrillo y de piedra. Siendo $D$ la matriz de elasticidad para un material isótropo, la matriz constitutiva $D^{\prime}$ es calculada como [3]:

$$
D^{\prime}=(1-d) D
$$

Siendo $r$ máx $\left\{r_{0}, r_{n}\right\}, A$ el parámetro que depende de la energía disipada por unidad de volumen para un proceso de tracción uniaxial, el índice de daño es [4]:

$$
d=1-\frac{r_{o}}{r} \exp \left\{A\left(1-\frac{r_{n}}{r_{o}}\right)\right\}
$$

\subsection{Acción sísmica}

Para analizar el comportamiento sísmico de la estructura se utiliza el método de análisis estático no lineal pushover o por empujes incrementales según el Eurocódigo 8 (21). Se trata de una técnica eficiente que permite estudiar la capacidad y la resistencia-deformación de la estructura bajo los efectos de un terremoto. La acción sísmica se introduce en la dirección de la estructura a analizar, sometiéndola a un patrón de cargas horizontales que se incrementan hasta alcanzar su capacidad máxima. En su aplicación se utiliza el espectro de aceleraciones para la ciudad de Valencia propuesto por la norma española NCSE-O2 (20). La intersección entre la curva de demanda y la de capacidad define el punto de desempeño o Performance Point. Este punto señala el movimiento que se puede esperar en el punto más elevado de la estructura para las acciones sísmicas de cálculo definido por el espectro.

A partir de la posición del punto de desempeño respecto a los puntos de cedencia y capacidad última se definen los umbrales de los estados de daño (Sd) (Figura 6).

Tabla 1. Clasificación de los materiales.

\begin{tabular}{|l|c|c|c|c|c|}
\hline \multicolumn{1}{|c|}{ Material } & $\begin{array}{c}\boldsymbol{D} \\
\left(\boldsymbol{t} / \mathbf{m}^{\mathbf{3}}\right)\end{array}$ & $\begin{array}{c}\boldsymbol{E} \\
\left(\mathbf{N} / \mathbf{m m}^{\mathbf{2}}\right)\end{array}$ & $\begin{array}{c}\boldsymbol{f c} \\
\left(\mathbf{N} / \mathbf{m m}^{\mathbf{2}}\right)\end{array}$ & $\begin{array}{c}\boldsymbol{f t} \\
\left(\mathbf{N} / \mathbf{m m}^{\mathbf{2}}\right)\end{array}$ & $\begin{array}{c}\boldsymbol{G f}^{+} \\
\left(\mathbf{N m m} / \mathbf{m m}^{\mathbf{2}}\right)\end{array}$ \\
\hline Sillería & 2,2 & 7.500 & 12 & 0,35 & 0,3 \\
\hline Relleno sillería & 2,0 & 2.500 & 8 & 0,25 & 0,2 \\
\hline Ladrillo & 1,8 & 3.500 & 3,5 & 0,15 & 0,1 \\
\hline Relleno bóveda & 1,5 & 1.000 & 3 & 0,2 & 0,15 \\
\hline
\end{tabular}




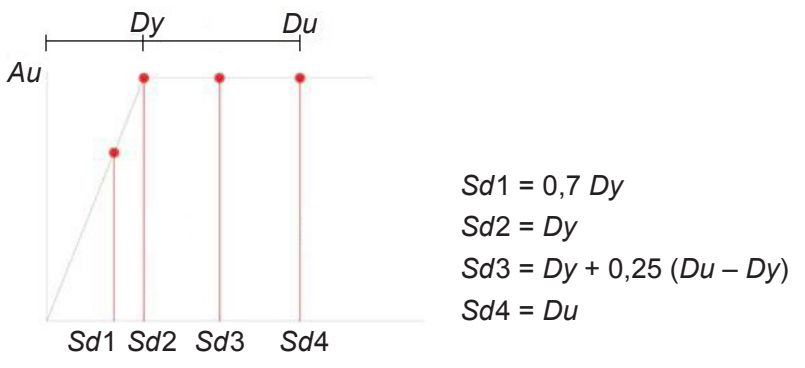

Figura 6. Umbrales de los estados de daño.

Para definir la acción sísmica se recurre a métodos de probabilidad. En este estudio se han utilizado dos eventos sísmicos, el espectro de diseño que corresponde a un período de retorno de 475 años y el sismo máximo con un período de retorno de 950 años. El período de retorno es una expresión que indica el promedio de tiempo en años que transcurre entre la ocurrencia de un seísmo que produce daños de una severidad igual o superior a una determinada.

\subsection{Modelo estructural}

El análisis se centra en el tramo central de la catedral, formado por las tres naves y dos crujías correspondientes al trazado primitivo. Éste es el tramo más vulnerable frente a acciones sísmicas, ya que en la dirección longitudinal del templo los empujes son absorbidos tramo a tramo y resistidos por la cabecera y los pies. El cálculo contempla cargas gravitatorias y acciones sísmicas transversales al edificio, actuando sobre este tramo central (22), (23).

Se elabora un modelo por medio de elementos finitos tipo «sólido» de unos $50 \mathrm{~cm}$ de tamaño medio, siguiendo los criterios de la denominada «macro-modelización», modelo más utilizado en el análisis de estructuras complejas (24). La estructura muraria y los pilares están formados por sillares careados por el exterior y con relleno de argamasa en su núcleo, por tanto los elementos utilizados modelizan la fábrica como un continuo, con las propiedades mecánicas homogeneizadas piedra-argamasa. Ello permite una menor exigencia de cálculo dada la enorme envergadura del objeto de estudio que contiene más de 16.500 elementos. Se ha considerado un terreno de tipo medio según especificaciones de la Norma Sismorresitente NCSE-02, que se ajusta al tipo del suelo de la catedral.
El análisis pushover permite evaluar la vulnerabilidad de la estructura y por tanto el modelo se aplica a la dirección transversal de la nave, por ser la más débil frente a sismo, por ello se modelan tres pórticos y se toman como valores de referencia los correspondientes al central.

A partir del estudio constructivo elaborado se plantean diferentes variaciones del modelo según los elementos constructivos considerados. El primer modelo reproduce la situación actual, incluyendo la losa de hormigón armado (construida en la intervención de 1976), además de los diafragmas y de los arbotantes, garantizando la continuidad de la estructura muraria. El segundo modelo de cálculo mantiene la losa de hormigón pero prescinde de los arbotantes manteniendo sólo los diafragmas, lo que nos permitirá comprobar su función estructural. En la tercera, cuarta y quinta simulación se elimina la losa de hormigón armado para analizar la estructura original de la catedral y se combina con y sin los arbotantes y diafragmas.

\section{RESULTADOS}

Con carácter general al tratarse de una estructura rígida, las propiedades dinámicas del modelo se sitúan en las zonas donde el espectro es más elevado y por tanto las aceleraciones efectivas son mayores. Para cargas gravitatorias las tensiones máximas se producen en la base de los pilares centrales alcanzando valores máximos de $3 \mathrm{~N} / \mathrm{mm}^{2}$ muy por debajo de la resistencia a compresión considerada en el cálculo del orden de $12 \mathrm{~N} / \mathrm{mm}^{2}$. El índice de daño se concentra en el intradós de las claves de los arcos advirtiéndose que los arbotantes superiores no colaboran al equilibrio de la estructura.

\subsection{Situación actual: modelo con losa, arbotantes y diafragmas}

En las gráficas se compara la capacidad de la estructura con la demanda provocada por el movimiento del terreno. La máxima deformación horizontal obtenida se corresponde con el punto de desempeño, situado en la intersección del espectro de capacidad de la estructura con la curva de demanda. Teniendo en cuenta un sismo con un período de retorno de 475 años, el punto de desempeño se sitúa previo a la fase elástica por lo tanto lejano al colapso (Figura 7a). En el sismo de 950 el punto de desempeño indica un nivel de daño medio-severo $(S d 3)$ según la tabla de clasificación de los umbrales de daño (Figura 7b).
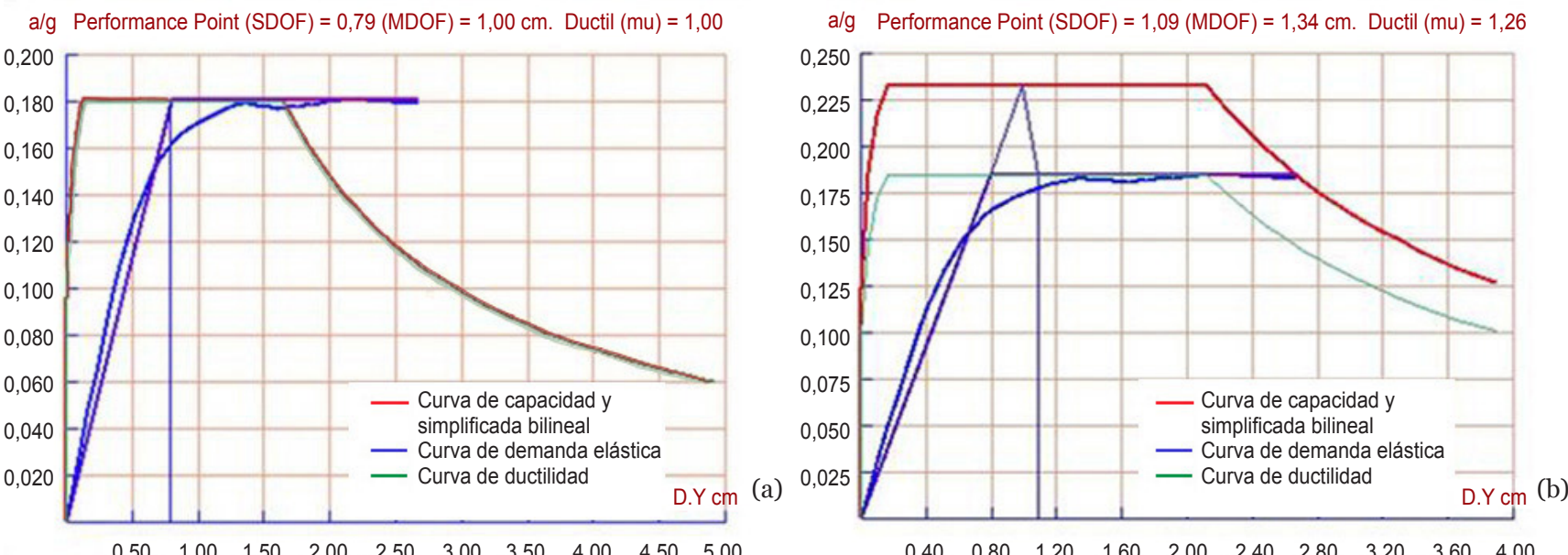

Figura 7. Determinación del punto de desempeño: a) sismo con tiempo de retorno de 475 años;

b) sismo con tiempo de retorno de 950 años. 


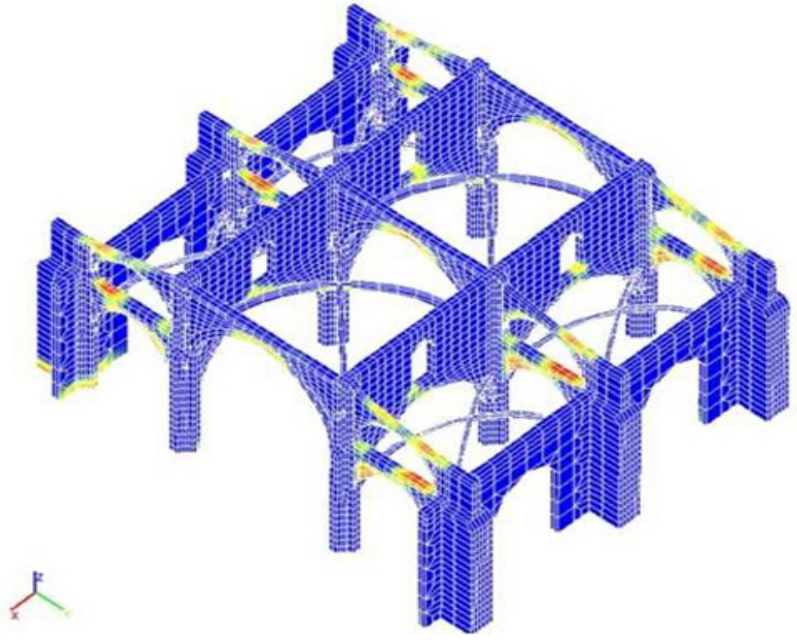

Figura 8. Índice de daño para el desplazamiento máximo.

En la Figura 8 el índice de daño se concentra en el intradós de los arcos y se observa como los contrafuertes entran en carga, aumenta el daño en el trasdós del arco justo donde se produce la disminución de su sección. Se alcanza la fisuración en las zonas indicadas, pero se trata de daños leves sin llegar al colapso global de la estructura.

\subsection{Función de los arbotantes: modelo con losa, diafragmas y sin arbotantes}

De nuevo para un sismo de 475 años el punto de desempeño se sitúa en el primer tramo de la fase inelástica (Figura 9a)

a/g Performance Point $(\mathrm{SDOF})=0,77(\mathrm{MDOF})=0,97 \mathrm{~cm}$. Ductil $(\mathrm{mu})=1,06$

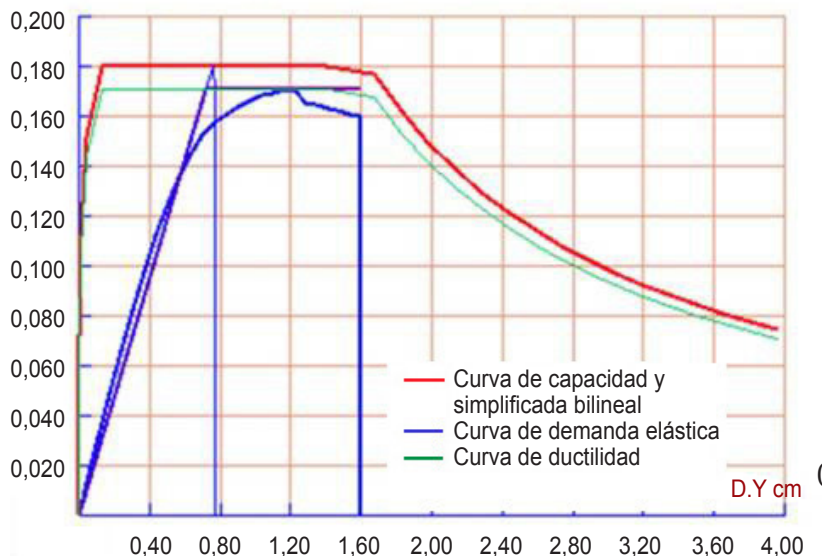

lo que supone daños leves. En el caso de un sismo con un período de retorno de 950 años el performance point se sitúa cercano al punto de rotura (Figura 9b) y por tanto los daños son graves.

Puede observarse que el daño se concentra en el intradós de los arcos de la nave central y trasdós de los arcos de las naves laterales (Figura 10). Aunque los daños son graves en estas zonas no se llega a alzanzar el colapso global.

\subsection{Situación original de la catedral: modelo sin losa, con diafragmas y con arbotantes}

En este caso, como muestra la Figura 11, la situación del punto de desempeño indica un nivel de daño medio severo $\left(S d_{3}\right)$ para un sismo con tiempo de retorno de 475 años. Al eliminar la losa del modelo el punto de rotura desciende, por tanto la estructura es más vulnerable. Los daños obtenidos se sitúan entre daños moderados y daños graves.

El cálculo realizado explica los daños históricos sufridos por la catedral. Como puede observarse en la Figura 12a el daño en arbotantes se concentra en zonas que han tenido que ser intervenidas y en arcos de las naves laterales (Figura 12b).

\subsection{Modelo sin losa, sin arbotantes y con diafragmas}

En la simulación en la que se elimina losa y no se consideran los arbotantes el punto de desempeño indica un nivel de daño completo (Sd4) (Figura 13a). Éstos se concentran

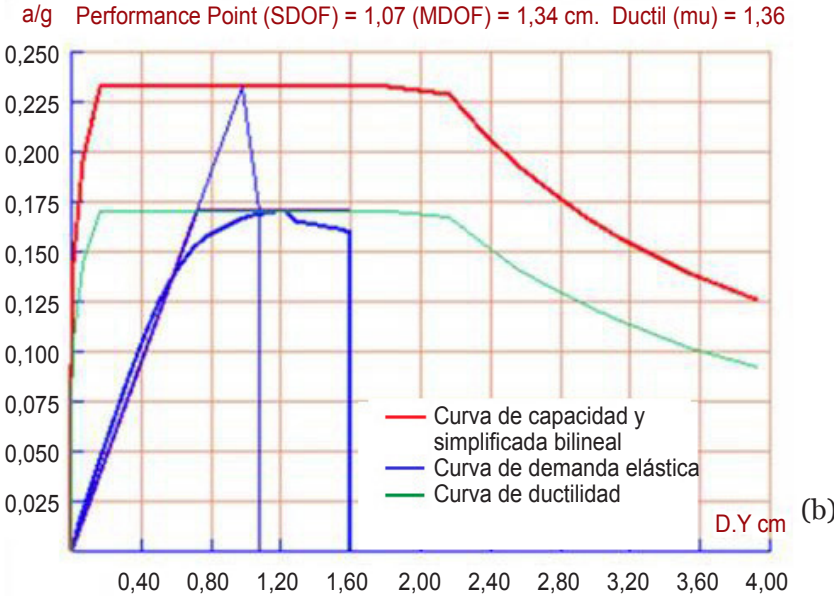

Figura 9. Determinación del punto de desempeño: a) sismo con tiempo de retorno de 475 años; b) sismo con tiempo de retorno de 950 años.
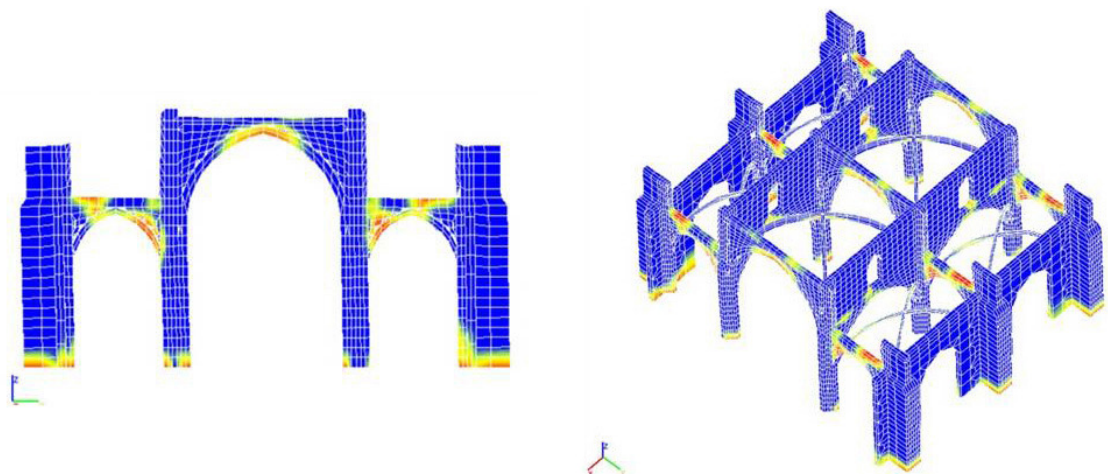

Figura 10. Índice de daño para el desplazamiento máximo para un sismo con tiempo de retorno de 950: a) alzado; b) perspectiva. 
a/g Performance Point $($ SDOF $)=0,74($ MDOF $)=0,93 \mathrm{~cm}$. Ductil $(\mathrm{mu})=1,15$

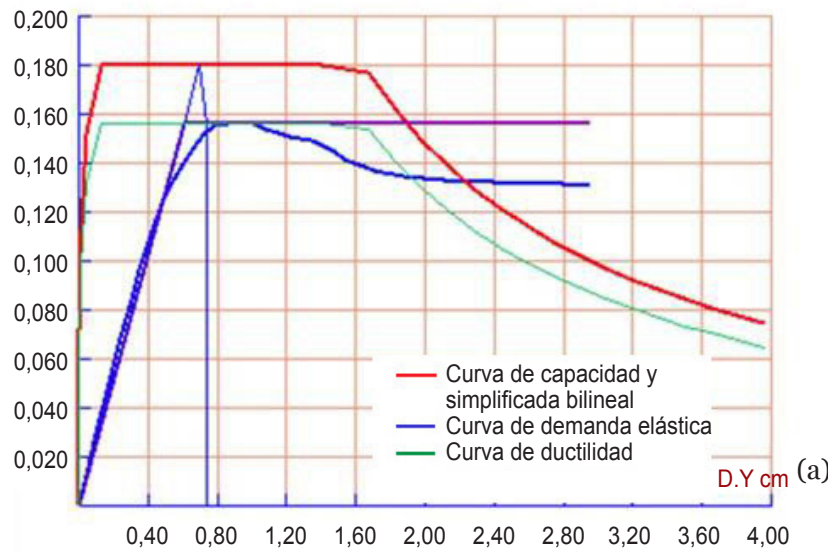

a/g Performance Point $(\mathrm{SDOF})=1,03(\mathrm{MDOF})=1,29 \mathrm{~cm}$. Ductil $(\mathrm{mu})=1,49$

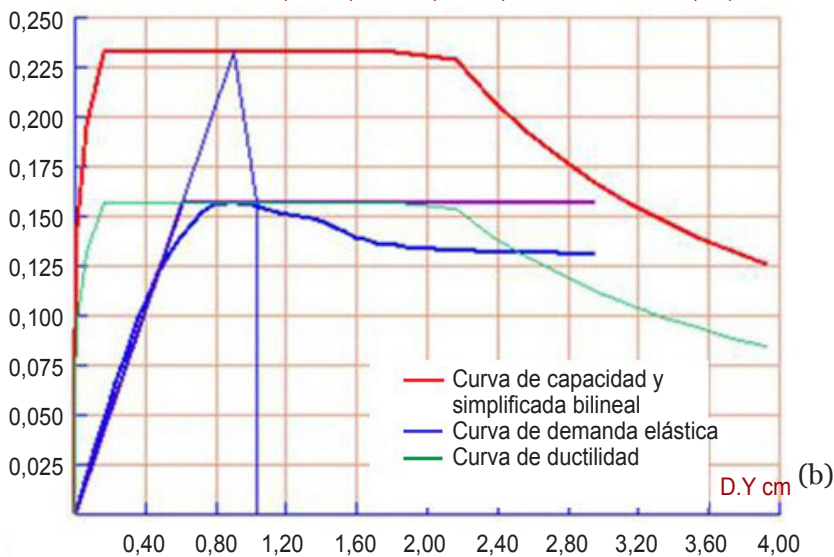

Figura 11. Determinación del punto de desempeño: a) sismo con tiempo de retorno de 475 años; b) sismo con tiempo de retorno de 950 años.

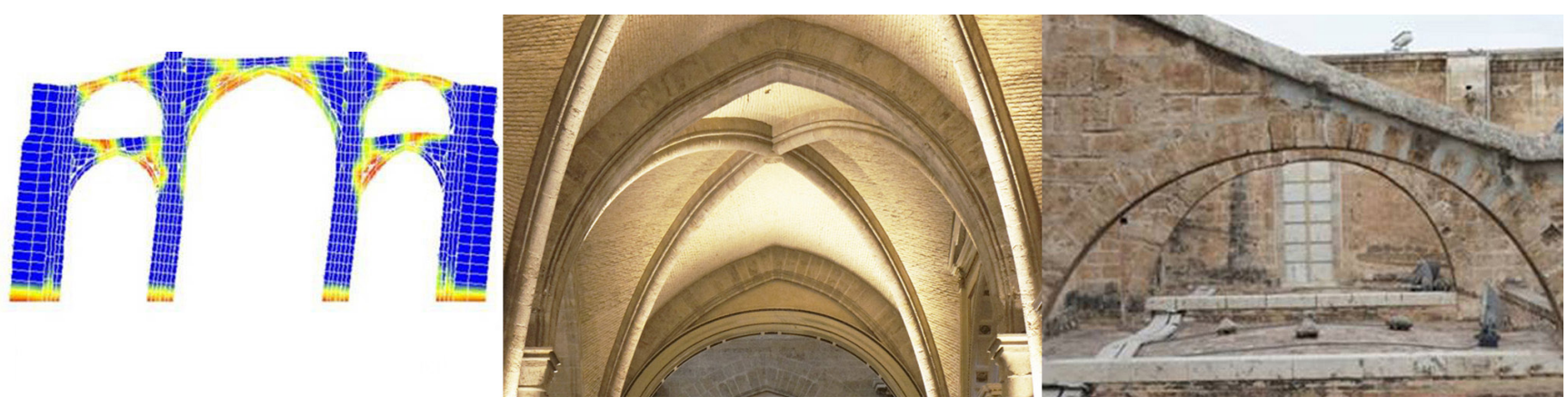

Figura 12. Índice de daño del desplazamiento máximo para un sismo con tiempo de retorno de 950 años: a) resultados sobre el modelo; b) daños observados en arcos fajones de las naves laterales y arbotantes.

en diafragmas extendiéndose en intradós de arcos y en los huecos de los cerramientos laterales de la nave principal (Figura 13b). Se producen daños graves llegando al colapso global para el escenario de sismo con tiempo de retorno de 950 años.

\subsection{Modelo sin losa, con arbotantes y sin diafragmas}

En este análisis se obtiene el valor más alto del punto de desempeño de todas las simulaciones realizadas (Figura 14a) lo que conduce a unos daños clasificados como graves que llegarían a producir el colapso. Al no considerar los diafragmas ni la losa, se pierde el concepto de caja, por lo que la capacidad sísmica transversal de la estructura comienza a ser insuficiente. Los desplazamientos son mayores como consecuencia de la pérdida de unidad del conjunto. El daño se concentra en arcos fajones y en la parte superior e inferior de los huecos de los muros formeros, alcanzándose el colapso de los arbotantes en la zona donde la sección del arco se reduce, por ser el punto más vulnerable frente a la rotura (Figura 14b). a/g Performance Point $(\mathrm{SDOF})=1,06(\mathrm{MDOF})=1,31 \mathrm{~cm}$. Ductil $(\mathrm{mu})=1,52$

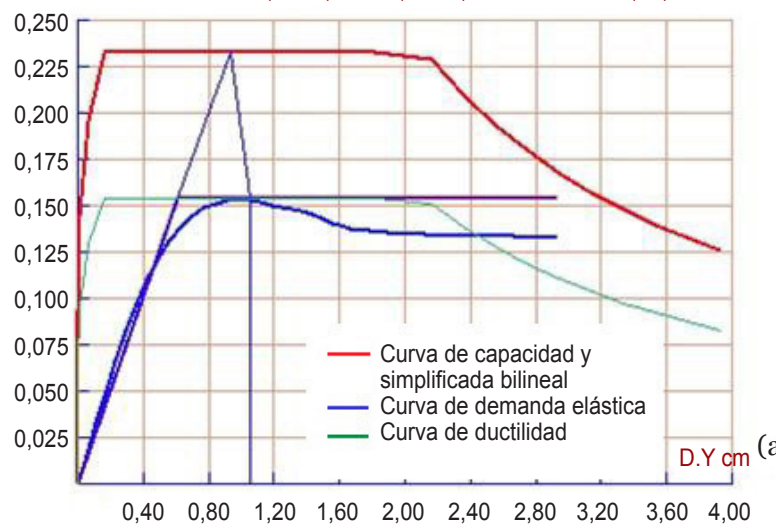

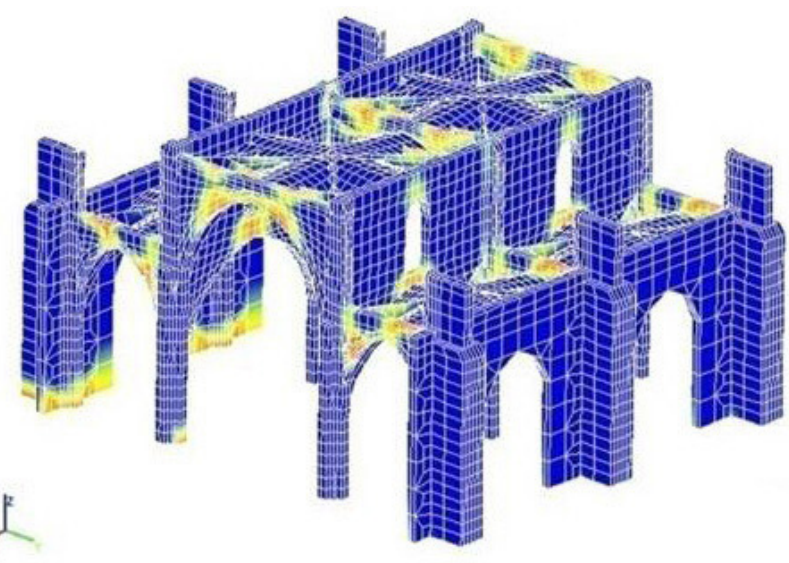

(b)

Figura 13. Sismo con un tiempo de retorno de 950 años: a) punto de desempeño; b) índice de daño. 
a/g Performance Point $(S D O F)=1,61(M D O F)=2,00 \mathrm{~cm}$. Ductil $(\mathrm{mu})=1,70$

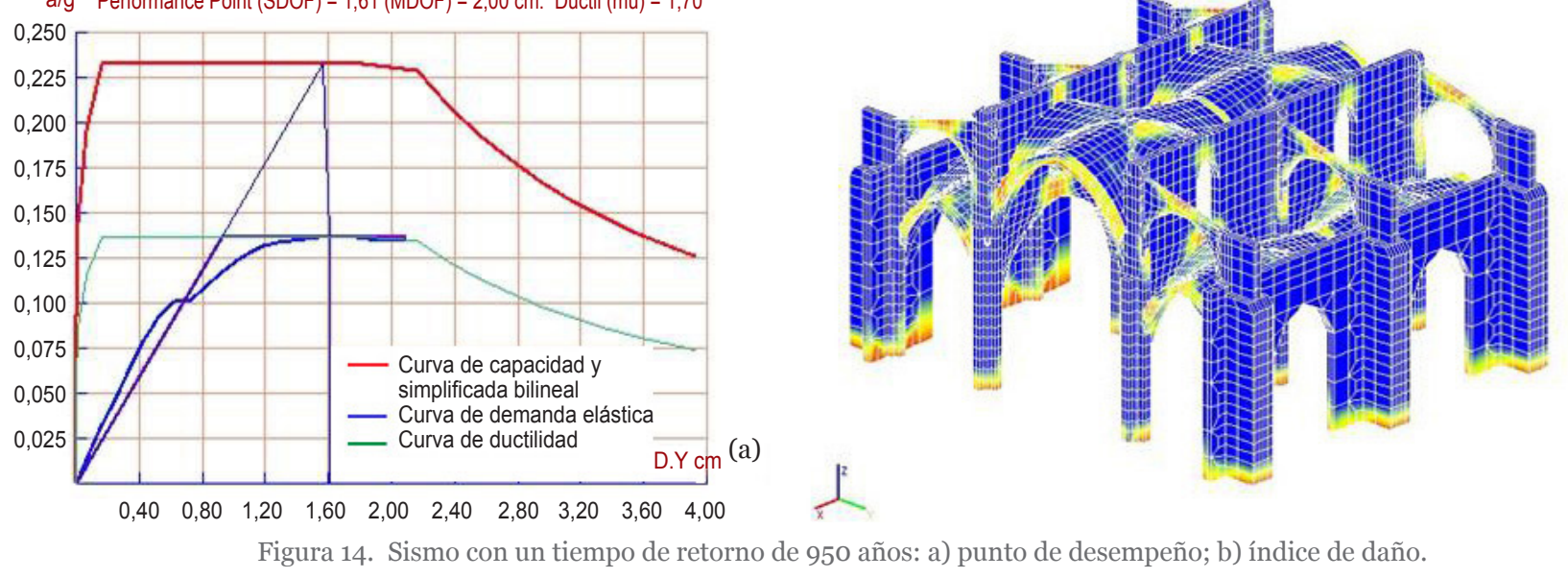

(b)

\section{CONCLUSIONES}

$\mathrm{Al}$ tratarse de una estructura de gran rigidez los períodos de oscilación obtenidos son bajos por lo que se sitúan en el espectro de demanda donde las aceleraciones son más elevadas.

Se ha comprobado que la capacidad sísmica de la catedral de Valencia, en su situación actual, es suficiente para soportar el terremoto con un período de retorno de 475 años. Los daños esperados, de producirse, serían leves. La situación empeora cuando se considera un terremoto con tiempo de retorno de 950 años. En este caso los daños principales se sitúan en el intradós de los arcos y se extiende en los diafragmas y arbotantes.

La eliminación de los arbotantes impide la continuidad en el sistema murario, perdiéndose la conexión entre los diafragmas y los contrafuertes. Los daños obtenidos son más graves, aunque no se llega al colapso global de la estructura. La simulación en la que se elimina la losa, pero se mantienen los diafragmas y los arbotantes que coincide con la situación primitiva de la catedral, reproduce los daños históricos sufridos y las deformaciones actuales en los arbotantes y arcos fajones. Cuando no se consideran ni los arbotantes ni la losa, el nivel de daños comienza a ser severo tanto en los diafragmas como en la parte superior e inferior de los huecos de los cerramientos de la nave central. En esta simulación se producen daños graves que conducirían al colapso. El modelo sin la losa ni los diafragmas representa la situación más vulnerable puesto que se pierde el sentido de la estructura muraria, produciéndose el colapso.

A la vista de los distintos resultados, es evidente que tanto el diafragma como los arbotantes son necesarios para el correcto comportamiento de la estructura de la catedral frente a un movimiento sísmico. No obstante hay que resaltar que la pérdida del diafragma resulta más determinante en el comportamiento del conjunto.

En aquellos modelos en los que se ha considerado la losa de hormigón armado de la cubierta se ha comprobado un aumento de la rigidez del conjunto. Frente a la opinión extendida de que un aumento de peso en altura supone una modificación negativa del comportamiento dinámico del edificio, en este caso supone un incremento de peso que es bajo en relación al peso total por tanto no modifica las propiedades dinámicas. Sin embargo sí que hay un aumento de la rigidez, atando los muros longitudinales y los diafragmas, favoreciendo el comportamiento frente a sismo.

Gracias a la forma y posición de los arbotantes, estos arcos se convierten en diafragmas que garantizan la continuidad con los contrafuertes y generan una estructura muraria que arriostra transversalmente, funcionando como «muros de cortante». Este entramado de muros ortogonales característico del «Gótico mediterráneo» dota a la estructura de la catedral de la rigidez y resistencia necesaria configurando la forma que mejor responde frente a movimientos sísmicos.

\section{REFERENCIAS}

(1) Heyman, J. (2004). Análisis de estructuras: un estudio histórico. Madrid: Reverte.

(2) Mira, E., Zaragozá, A. (2003). Una arquitectura gótica mediterránea. Valencia: Conselleria de Cultura i Educació.

(3) Cassinello, M. (2004). Trazado y estabilidad de la arquitectura gótica. Cuadernos Intemac, 53

(4) Medina, J.M. (2014). Clasificación inédita de catedrales góticas españolas en función de su iluminación. Informes de la Construcción, 66(535): e028, doi: http://dx.doi.org/10.3989/ic.12.132.

(5) Cassinello, P. (2005). Influencia de los terremotos históricos en la construcción de las catedrales góticas españolas. Annali Di Architettura: Rivista del centro Internazionale di Studi di Architettura “Andrea Palladio" (17): 9-20.

(6) Roca, P., Cervera, M., Pelà, L., Clemente, R., Chiumenti, M. (2013). Continuum FE Models for the Analysis of Mallorca Cathedral. Engineering Structures, 46: 653-670, doi: http://dx.doi.org/10.1016/j.engstruct.2012.08.005.

(7) ANGLE. (2014). Structural Analysis Software for Finite Element. Developed by A. Alonso. Department of Mechanics of the Continuous Medium and Theory of Structures. Universitat Politècnica de València, Spain.

(8) Marques, R., Lourenço, P.B. (2013). A model for pushover analysis of confined masonry structures: Implementation and validation. Bulletin of Earthquake Engineering, 11(6): 2133-2150, doi: http://dx.doi.org/10.1007/s10518-013-9497-5. 
(9) Oñate, E., Hanganu, A., Barbat, A. (1996). Structural Analysis and Durability Assessment of Historical Constructions using a Finite Damage Model. Barcelona: CIMNE.

(10) Zaragozá Catalán, A. (1990). Iglesias de arcos diafragma y armadura de madera en la arquitectura medieval valenciana. Valencia: Universidad Politécnica de Valencia.

(11) Cassinello, P. (2005, 27 de enero). Racionalidad sísmica en la arquitectura ojival: tipos estructurales y constructivos. En Cuarto Congreso Nacional de Historia de la Construcción, pp. 249-258. Cádiz: Instituto Juan de Herrera.

(12) Casinello, P., Álvarez, M., Rodríguez, R., Miranda, M., Ramos, L.A., Azorín, V. (2014). Levantamiento del comedor del IETcc: una crónica de la innovación en la representación de edificios. Informes de la Construcción, 66(536): e039, doi: http://dx.doi.org/10.3989/ic.14.023.

(13) Vargas, Y.F., Pujades, L.G., Barbat, A.H., Hurtado, J.E. (2013). Incremental Dynamic Analysis and Pushover Analysis. A Probabilistic Comparison. Computational Methods in Applied Sciences, 26: 293-308, doi: http://dx.doi.org/10.1007/97894-007-5134-7_17.

(14) Ramos, A., León, J. (2013). Classification of backfill at the extrados of masonry vaults. Informes de la Construccion, 65(532): 471-480, doi: http://dx.doi.org/10.3989/ic.12.062.

(15) Orduña, A., Roeder, G., Peña, F. (2007). Evaluación sísmica de construcciones históricas de mampostería: comparación de tres modelos de análisis. Revista de Ingeniería Sísmica, 77: 71-88.

(16) Navarro, A., Lizondo, L., Martínez, A., Alonso, A. (2009, 21 de octubre). Origen, influencias y realidad en la construcción del puente de Trinidad de Valencia. En Congreso Nacional de Historia de la Construcción, pp. 989-1002. Valencia.

(17) Alonso, A., Martínez, A., Llopis, V., Moreno, J. (2012) Structural Behaviour Analysis of the Cathedral of Valencia. Structural Analysis of Historical Constructions. En SAHC 2012 8th International Conference on Structural Analysis of Historical Constructions, pp. 185-192. Poland.

(18) Lluis i Ginovart, J., Costa A., Fortuny, G., Solà-Morales, P., Toldrà, J.M. (2013). Proceso de rotura de una cúpula oval en la catedral de Tortosa. Análisis del mecanismo de colapso. Informes de la Construcción, 65(532): 509-517, doi: http:// dx.doi.org/10.3989/ic.12.069.

(19) Oller, S. (2001). Fractura mecánica. Un enfoque global. Barcelona: CIMNE.

(20) NCSE-02. (2002). Real Decreto 997/2002, de 27 de septiembre, por el que se aprueba la Norma de Construcción Sismoresistente (NCSE-02). Boletín Oficial del Estado (244): 35898-35967. España: Ministerio de Fomento.

(21) EC-8 (2004). ENV 1998-4:2004 Eurocode 8: Design of structures for earthquake resistence. Part1: General rules, seismic actions and rules for buildings.

(22) Irizarry, J., Podestà, S., Resemini, S. (2003, 1-4 de abril). Curvas de capacidad para edificios monumentales: la iglesia Santa María del Mar de Barcelona. En Segundo Congreso Nacional de Ingeniería Sísmica, pp. 541-555. Málaga.

(23) Vacas, A. (2009). Análisis sísmico de las catedrales góticas mediante el método del espectro de capacidad. Universidad Politécnica de Cataluña.

(24) Roca, P., Cervera, M., Gariup, G., Pela, L. (2010). Structural Analysis of Masonry Historical Constructions. Classical and Advanced Approaches. Archives of Computational Methods in Engineering, 17: 299-325, doi: http://dx.doi. org/10.1007/s11831-010-9046-1. 